\title{
Evaluation of the hepatoprotective effect of silver nanoparticles in acetaminophen induced hepatic injury in albino rats
}

\author{
Mohd Salim Reshi* and Sangeeta Shukla \\ UNESCO- Trace Element Satellite Center \\ School of Studies in Zoology, Jiwaji University, Gwalior (M.P). India \\ *Email: reshisalim@gmail.com
}

Silver is one of the heavy metals and have a long history (over 6000 years) as a medicinal drug. Silver nanoparticles (AgNPs) exhibits remarkable physical, chemical and biological properties such as antimicrobial effects due to its unique mechanism of action. AgNPs were used widely as an antibiotic against wound infections and serious burn injuries. The purpose of this study was to evaluate the protective effect of AgNPs on Acetaminophen (APAP) induced hepatic injury in rats. Female albino rats of Wistar strain were selected for the present study and were divided into various groups of six animals each. Animals were administered with APAP at a dose of $20 \mathrm{mg} / \mathrm{kg}$ p.o (5 days/week for 4 weeks). Animals were treated with AgNPs at a dose of $100 \mu \mathrm{g} / \mathrm{kg}$ p.o. and silymarin at a dose of 50 $\mathrm{mg} / \mathrm{kg}$ p.o. for 2 days/week for 4 weeks. AgNPs showed a remarkable protective and antioxidant activity against APAP induced toxicity as judged from the serum marker enzymes and antioxidant levels in liver tissues. APAP induced a significant rise in aspartate amino transferase (AST), alanine amino transferase (ALT), lactate dehydrogenase (LDH), serum alkaline phosphatase (SALP) and total bilirubin, which indicated the hepatocellular damage. Oxidative stress was observed by increased Lipid peroxidation (LPO) with a reduction of reduced glutathione (GSH) content after toxicant administration. Activity of major antioxidant enzymes, superoxide dismutase (SOD), catalase (CAT) were significantly inhibited by APAP intoxication. GSH cycle enzymes, viz. glutathione reductase (GR) and glutathione peroxidase (GPx) were significantly declined due to exposure of APAP. APAP intoxication was also found to induce DNA damage which was assessed by comet assay. The damage was expressed in terms of percent DNA damage, tail length and tail moment. Treatment of rats with AgNPs significantly restored serum marker enzymes towards normal which indicates the repair of hepatic membrane by the treatment of AgNPs. AgNP showed remarkable antioxidant property which was evident from the reduced
LPO and maintenance of GSH level towards normal in liver. Activities of free radical scavenging enzymes like SOD and CAT were significantly recovered near to normal by the treatment of AgNPs. AgNPs significantly restored the activities of GR and GPx, hence maintained the homeostasis of GSH. AgNPs significantly protected the DNA damage which was confirmed by the depletion in tail length, tail moment and percent DNA damage. This protection of DNA damage by AgNPs may be due to the inhibition of reactive oxygen species (ROS) by the AgNPs. Our biochemical investigations were also supported by histological studies. Transmission electron microscopy of liver of rat intoxicated with APAP showed deterioration of nuclear envelope (NE), degeneration of mitochondria (M) and degranulation of endoplasmic reticulum (ER). AgNPs treatment significantly showed the regeneration in the cellular organelles towards normal. The efficacy of AgNPs were comparable to the standard drug silymarin, data indicated a positive effect. Thus it is concluded that AgNPs showed remarkable ameliorative effect against APAP induced toxicity. Hence after adequate study AgNPs may be used for the development of a drug against hepatic diseases to raise the hope and expectations of patients with liver diseases. 